\title{
X-RAY POWDER DIFFRACTION AS A TOOL TO STRUCTURE DETERMINATION OF LASSBIO-1755
}

\author{
Bastos, I. T. S. ${ }^{1}{ }^{*}$; Costa, F. N. ${ }^{2}$; da Silva, T. F. ${ }^{3}$; Barreiro, E. J. ${ }^{3}$; Lima, L. M. ${ }^{1}$; Fer- \\ reira, F. F. ${ }^{2}$; Braz, D. ${ }^{4}$; Barroso, R. C. ${ }^{1}$ \\ ${ }^{1}$ Instituto de Física, Universidade do Estado do Rio de Janeiro (UERJ), Rio de Janeiro, RJ, Brazil \\ ${ }^{2}$ Centro de Ciências Naturais e Humanas (CCNH), Universidade Federal do ABC (UFABC); Santo André, SP, Brazil \\ ${ }^{3}$ Laboratório de Avaliação e Síntese de Substâncias Bioativas (LASSBio), Faculdade de Farmácia, Universidade Federal do Rio de Janeiro, \\ Rio de Janeiro, RJ, Brazil \\ ${ }^{4}$ Laboratório de Instrumentação Nuclear/COPPE, Universidade Federal do Rio de Janeiro, Rio de Janeiro, RJ, Brazil
}

*isadoratairinne@gmail.com

\begin{abstract}
New substances are constantly being synthesized and many ancient structures are still unknown. As important as the design and synthesis of new drugs is their structural characterization, since its structure (conformation) can be directly related to the therapeutic action. The use of X-ray diffraction was and has been very important in structural determination of new compounds synthesized. In this work was done the structure determination of LASSBio- 1755 with $\mathrm{X}$-ray powder diffraction data, a $\mathrm{N}$-acylhydrazone (NAH) derivative lead-compound, synthesized in Laboratory of Synthesis and Evaluation of Bioactive Substances $\left(\operatorname{LASSBio}^{\circledR}\right)$ of the Federal University do Rio de Janeiro. The NAH moiety is minimal subunit present in the various compounds prototype drugs capable of producing interaction points with more than one type of bioreceptor. This compound crystallizes in a triclinic (P-1) space group, with unit cell parameters $a=4,86647(9) \AA, b=9,3108(2) \AA, c=11,3402(2) \AA, \alpha=106,6489(13)^{\circ}, \beta=101,9581(12)^{\circ}, \gamma=$ $82,6286(16)^{\circ}, V=480,302(18) \AA^{3}$. The crystal structure of LASSBio- 1755 consists of two molecules in unit cell ( $Z$ $=2$ ), accommodating one molecule in the asymmetric unit $\left(Z^{\prime}=1\right)$. Rietveld method was used to refine the crystal structure and the goodness-of-fit indicator were $\chi^{2}=1.131$ and $\mathrm{R}_{\mathrm{wp}}=4.174 \%$.
\end{abstract}

